\title{
Über die Darstellung von Vanadin und anderen Metallen nach dem Thermitverfahren.
}

\author{
Von \\ Wilhelm Prandta und Benno Bleyer.
}

Wir sind seit längerer Zeit mit den Vorarbeiten zu einer Neubestimmung des Atomgewichtes des Vanadins beschäftigt und benötigten zur Darstellung sauerstofffreier Vanadinverbindungen grölserer Mengen elementaren Vanadins. Als Ausgangsmaterial für dessen Darstellung kommt in letzter Linie das käufliche Ammoniummetavanadat, $\mathrm{NH}_{4} \mathrm{VO}_{3}$, bzw. das daraus durch einfaches Rösten darstellbare Vanadimpentoxyd, $\mathrm{V}_{2} \mathrm{O}_{5}$, in Betracht. Es ist bekannt, dafs das Vanadinpentoxyd einen Teil seines Sauerstoffs leicht, den Rest aber erst unter dem Einflufs der allerstärksten Reduktionsmittel abgibt; die Darstellung des Vanadinmetalles gilt deshalb mit Recht als eine besonders schwierige Aufgabe.

So ist es z. B. Moissan, ${ }^{1}$ Helouis, ${ }^{2}$ Goldschmidt ${ }^{3}$ und Koppel und $\mathrm{K}_{\triangle \mathrm{UFMANN}}{ }^{4}$ nicht gelungen, das Vanadinpentoxyd durch Aluminium zu Metall zu reduzieren. Letztere erhielten zwar einen metallisch aussehenden Regulus, der indes nur ca. 80\% Vanadin enthielt und vermutlich aus einem Gemenge von Vanadin und niederen Vanadinoxyden bestand; der von GoLdschmide erschmolzene Regulus war nach HiтtoRF Vanadinoxydul $\nabla_{2} O$. Neuerdings haben zwar VogeL und TAMmanN ${ }^{5}$ Vanadin auf aluminothermischem Wege dargestellt; wir konnten aber auf diesem Wege ebensowenig wie die früher genannten Forscher ein einigermalsen reines Metall erhalten. Schon früher hatte Morssax ${ }^{B}$ versucht, das Vanadinpentoxyd mittels Kohle im elektrischen Ofen zu Vanadin zu reduzieren, aber schliels-

1 Compt. rend. 122 (1896), 1297.

2 Bull. Soc. Encouragement 1896, 904.

${ }^{3}$ Z. f. Elektrochem. 6 (1899-1900), 53.

4 $Z$. anorg. Chem. 4อ̃ (1905), 352.

8 Z. anorg. Chem. 58 (1908), 73.

${ }^{6}$ Compt. rend. 116 (1893), 1225; 122 (1896), 1297.

Z. anorg. Chem. Bd. 61. 
lich nur ein mehr oder weniger carbidhaltiges Metall erhalten. Dagegen stellten Muthmann und seine Mitarbeiter' angeblich ganz reines metallisches Vanadin dar, indem sie Vanadinpentoxyd mittels sogen. Mischmetalles, einer Legierung sämtlicher Cerit- und Yttermetalle, reduzierten. Das Mischmetall ist aber nicht nur nicht im Handel erhältlich, es ist auch seine Darstellung im Laboratorium sehr umständlich und kostspielig. Zur Gewinnung gröfserer Mengen Vanadin kann deshalb das Muthmannsche Verfahren nicht in Betracht kommen; überdies ist das dadurch erhaltene Vanadin nicht so rein, wie Weiss und Archel ${ }^{2}$ angeben. Herr Professor Muthmann hatte die Liebenswürdigkeit, uns eine Probe des von seinen Mitarbeitern dargestellten Vanadins zur Analyse zu überlassen; wir fanden darin nur gegen $91-93 \%$ Vanadin.

Zur Analyse wurden $0.5077 \mathrm{~g}$ blanke, schlackenfreie Splitter des Metalls in einer Platinschale in Salpersäure gelöst, die Lösung verdampft, der Rückstand geröstet und schliefslich zum Schmelzen erhitzt. Das so erhaltene Vanadinpentoxyd - $0.8676 \mathrm{~g}$ - verriet schon dureh sein Aussehen, dafs es uicht rein war. Während reines Vanadinpentoxyd in gelbbraunen durehsichtigen langen Prismen krystallisiert, war das aus dem Metall erhaltene dunkelbraun und undeutlich krystallinisch, zum Teil sogar glasig erstarrt; beim Erkalten sprang es in kleinen Splittern knisternd von der Platinschale ab, was reines Vanadinpentoxyd nie tut. In Ammoniakwasser löste es sich nicht klar auf; es blieben gelblichweifse Flocken (von Kieselsäure oder Erden) in nennenswerter Menge ungelöst zurück. ${ }^{3} \mathrm{E}_{\mathrm{s}}$ wurde deshalb der Vanadingehalt noch titrimetrisch mit Kaliumpermangariat bestimmt. Das $0.8676 \mathrm{~g}$ betragende Oxydationsprodukt wurde in wenig konzentrierter Schwefelsäure gelöst, die Lösung nach dem Verdünnen mit Wasser durch schweflige Säure reduziert und in der bekannten Weise mit Permanganat wiederholt titriert. Sie verbrauchte $102.85 \mathrm{ccm}$ einer gegen reines Vanadinpentoxyd eingestellten Kaliumpermanganatlösung, von der $1 \mathrm{ccm} 0.00815 \mathrm{~g} \mathrm{~V}_{2} \mathrm{O}_{5}$ oder $0.004575 \mathrm{~g} \mathrm{~V}$ entsprach. Das Oxydationsprodukt des von Weiss und Aicher dargestellten Metalls enthielt also nur $96.6 \% \quad \mathrm{~V}_{2} \mathrm{O}_{5}$ und das Metall selbst nur 92.7\% Vanadin. - Bei zwei weiteren Versuchen wurden $0.1470 \mathrm{~g}$ und $0.1800 \mathrm{~g}$ des Metalls unter Zusatz von etwas Salpetersäure in Schwefelsäure gelöst; nach dem Vertreiben der überschüssigen Salpetersäure wurden die Lösungen verdünnt, mit schwefliger Säure reduziert und mit der obigen Kaliumpermanganatlösung titriert. Sie verbrauchten 29.0 und $35.8 \mathrm{~cm}$ Permanganatlösung; die beiden Proben enthielten also 90.25 und $91.0 \%$ Vanadin.

1 Chem.-Ztg. 28 (1904), 506; Ann. Chem. 337 (1904), 380; 355 (1907), 58.

2 Ann. Chem. 337 (1904), 380.

${ }^{3}$ Das Verhalten gegen wässeriges Ammoniak wurde nicht an der zu obiger Analyse verwendeten Probe, sondern an einer anderen geprüft. 
Das Verfahren von Roscoe, ${ }^{1}$ der sauerstofffreie Vanadinverbindungen durch Glühen im Wasserstoffstrome oder mittels metallischen Natriums reduzierte, liefert zwar ein ziemlich reines Vanadinmetall, aber mit so grofsem Aufwande und in so geringen Mengen, dals es für unsere $Z$ wecke nicht anwendbar ist.

Wir suchten deshalb nach einer neuen Methode, welche ohne grofse Kosten grölsere Mengen Vanadinmetall liefern sollte. Es scheint bisher noch nicht versucht worden zu sein, Vanadin durch Reduktion seiner Oxyde mittels Calciums darzustellen. Das Calcium ist unter den Bedingungen des GoLDschurd schen aluminothermischen Verfahrens ein äufserst kräftiges Reduktionsmittel. Seine Verwendbarkeit ist aber dadurch beschränkt, dafs das bei der Reduktion entstehende Calciumoxyd bei der Reaktionstemperatur noch nicht flüssig wird; es kann sich deshalb das entstandene Metall nicht von der Schlacke sondern und man erhält im allgemeinen gesinterte, krümelige Schlackenmassen, die das jeweils reduzierte Metall in sehr feiner Verteilung enthalten. GoLDsCHMIDT ${ }^{2}$ suchte diesem Übelstande dadurch abzuhelfen, dafs er statt des reinen Calciums Gemenge desselben mit Silicium oder Aluminium anwendet; man erhält dann leichter flüssige Calciumsilicat- bzw. Calciumaluminatschlacken.

Wie unsere Versuche ergaben, kann metallisches Calcium allein wegen der hohen Schmelztemperatur seines Oxyds auch zur Darstellung von Vanadin aus Vanadinpentoxyd nicht verwendet werden. Vanadinpentoxyd wird durch Calcium in einer sehr heftigen Reaktion wohl zu Metall reduziert, aber das Metall ist in Gestalt sehr kleiner Kügelchen in der ganzen Schlackenmasse verteilt. Es ist also auch hier notwendig, Zusätze zu machen, welche die Bildung einer leichter schmelzbaren Schlacke veranlassen. Zusätze von Calciumfluorid oder Kryolith hatten nicht den gewünschten Erfolg. Da Vanadin sich mit Silicium sehr leicht zu ziemlich beständigen Siliciden verbindet, kann man von vorn herein nicht erwarten, durch Reduktion von Vanadinpentoxyd mit Calcium-Siliciumgemengen ein auch nur annähernd reines Metall zu erhalten; wir haben deshalb in dieser Richtung gar keine Versuche angestellt. Dagegen haben wir bei der Verwendung von Calcium-Aluminumgemengen aus Vanadinpentoxyd ein Metall erhalten, das zwar nicht ganz reines Vanadin ist,

I Ann. Chem. Pharm., Suppl. 7 (1870), 70; vgl. auch Setrerbera, Öfvers. K. Vet.-Akad. Förhandl. 39 (1882), Nr. 10, 13.

2 Z. f. Elektrochem. 14 (1908), 558. 
das im Vanadingehalt aber dem nach dem Muthmannschen Verfahren erhaltenen Metall mindestens gleichkommt und ein geeignetes und verhältnismälsig billiges Ausgangsmaterial für die Darstellung sauerstofffreier Vanadinverbindungen bildet. GoLDschмmт (a. a. O.) empfiehlt besonders Gemenge von 3 Atomen $\mathrm{Ca}+4$ Atomen $\mathrm{Al}(53+$ 47 Gewichtsteilen) und 1 Atom $\mathrm{Ca}+1$ Atom Al $(63+37$ Gewichtsteilen), welche besonders leicht (bei ca. $1400^{\circ}$ ) schmelzende Calciumaluminatschlacken von der Zusammensetzung $3 \mathrm{CaO} .2 \mathrm{Al}_{2} \mathrm{O}_{3}$ bzw. $2 \mathrm{CaO} . \mathrm{Al}_{2} \mathrm{O}_{3}$ bilden. Wir haben gefunden, dafs bei der Reduktion von Vanadinpentoxyd ein Gemenge ron 3 Atomen $\mathrm{Ca}+2$ Atomen $\mathrm{Al}$ $(69+31$ Gewichtsteilen) noch energischer wirkt und eine gut durchgeschmolzene, homogene, krystallinische Schlacke und einen blasenfreien Regulus liefert. Die bei der Reduktion von Vanadinpentoxyd mit Calcium-Aluminiumgemischen freiwerdende Wärme reicht nicht nur aus, die Reaktionsprodukte vollkommen zu schmelzen, man kann dem Gemenge auch früher erhaltenes Vanadinmetall grob gepulvert in ziemlich beträchtlichen Mengen zum Umschmelzen zusetzen. Wir haben dem $\mathrm{Ca}-\mathrm{Al}-\mathrm{V}_{2} \mathrm{O}_{5}$-Gemenge wiederholt auch vanadinhaltige Schlacken mit anhaftenden Metallstücken von früheren Versuchen zugesetzt; durch solche Zuschläge lälst sich nicht nur die Heftigkeit der Reaktion beliebig herabmindern, man kann damit zugleich die Ausbeute an Metall verbessern. Notwendig sind derartige Zusätze, wenn man erheblich mehr als $100 \mathrm{~g}_{2} \mathrm{O}_{5}$ in einem Versuch reduziert, weil sonst die Reaktion so heftig wird, dafs ein grolser Teil der gesrhmolzenen Massen verschleudert wird.

Das mit Hilfe von Calcium-Aluminiumthermit erbaltene Vanadin ist ein hellstahlgraues, sehr hartes, politurfähiges Metall. Es ritzt Glas, Stahl und Quarz und lälst sich mit dem Hammer nur schwierig zerschlagen, im Gegensatz $z \mathfrak{u}$ dem ron WeIss und AICHEL erhaltenen Metall, welches unter dem Hammer wie Glas zerspringt. Der Bruch ist fein- bis grobkrystallinisch. Am Carborundrad geschliffen und poliert, bleibt es auch nach wochenlangem Liegen in der Laboratoriumsluft vollkommen blank. Zur Befreiung von anhaftender Schlacke kann man das Metall mit konzentrierter Salzsäure kochen. Trotz oft wiederholter Versuche ist es uns nicht gelungen, ein Metali mit mehr als $94.5 \%$ Vanadin zu erzielen; es enthielt stets geringe Mengen Kohlenstoff, Silicium, Aluminium und Eisen, die aus dem verwendeten käuflichen Calcium und Aluminium stammen. Die Schwierigkeit, ganz reines Vanadin zu erbalten, besteht vor allem darin, dafs das Vanadin sich nicht nur mit Sauerstoff, sondern 
auch mit Kohlenstoff und Silicium sehr leicht zu Verbindungen vereinigt, die gegen Oxydationsmittel viel beständiger sind als das reine Vanadin. Das Vanadinmetall läfst sich deshalb auch nicht durch Umschmelzen reinigen; es wird im Gegenteil dabei immer unreiner, da zuerst Vanadin verschlackt wird, während die Verunreinigungen sich anreichern. Man kann nach dem Thermitverfahren reines Vanadin nur erhalten, wenn man ganz reines Calcium und Aluminium verwendet. Das gegenwärtig käufliche Calcium ist aber stark verunreinigt, vor allem mit Calciumcarbid, Calciumsilicid usw.

Bei der Darstellung von Vanadin und von anderen Metallen, die sich mit Silicium leicht zu Siliciden vereinigen, ist man gezwungen, die Thermitreaktionen in kieselsäurefreien Gefäfsen auszuführen. Als solche dienen Magnesiatiegel und Eisentiegel oder hessische Tiegel mit Magnesiaausfütterung. Derartige Tiegel sind ziemlich teuer und werden gewöhnlich schon beim ersten Versuch vollständig zerrissen. Ihre Verwendung bedeutet deshalb eine beträchtliche Verteuerung des Thermitverfahrens. Wir haben uns nun mit dem besten Erfolge von der Anwendung nicht nur der Magnesiatiegel, sondern überhaupt jedes Tiegels unabhängig gemacht, und da wir glauben, dafs unsere Versuchsanordnung allen von Nutzen sein wird, die durch Thermitreaktionen Metalle erzeugen wollen, werden wir sie näher beschreiben. Als Gefälsmaterial verwendeten wir bei allen im folgenden wiedergegebenen Versuchen feingemahlenen Flufsspat. In eine beliebige Blechbüchse - wir verwendeten zylindrische Blechbüchsen von $20-25 \mathrm{~cm}$ Höhe und ungefähr $12 \mathrm{~cm}$ Durchmesser, die als Emballagen gedient hatten - schüttet man eine 3-4 cm dicke Schicht Flulsspatmehl und stampft es fest; dann setzt man in die Mitte der Büchse ein beiderseits offenes zylindrisches Rohr - wir verwendeten einen der bekannten Zylinder für Gasglühlicht aus Jenaer Glas von etwa $25 \mathrm{~cm}$ Länge und ungefähr $4.5 \mathrm{~cm}$ Durchmesser - und füllt den Zwischenraum zwischen dem Zylinder und der Blechbüchse mit Flufsspatpulver aus, das man möglichst fest einstampft. In den durch den Zylinder freigehaltenen Schacht füllt man auf die $3-4 \mathrm{~cm}$ starke Flufsspatunterlage das Thermitgemenge ein, stampft es fest und zieht gleichzeitig den Zylinder unter drehenden Bewegungen langsam heraus. Wir wählten die Tiefe des Schachtes stets so, dafs die Oberfläche des Thermitgemenges mindestens einige Zentimeter unter dem oberen Schachtrande lag. Man entzündet dann den Schachtinhalt mittels einer. 
Zündkirsche; ${ }^{1}$ die Reaktion verläuft in dem elastischen Flufsspatschachte viel besser als in festen Tiegeln, es wird nur wenig Schlacke ausgeworfen, der Flufsspat kommt kaum ins Schmelzen. Nach Beendigung der Reaktion läfst man das Reaktionsgemenge in der Blechbüchse etwas erkalten, holt dann den mit der Schlacke und etwas Flufsspat zu einem Klumpen vereinigten Regulus mit einer Zange heraus, schreckt ihn mit kaltem Wasser ab und trennt ihn dann durch einige Hammerschläge von der Schlackenmasse. Der Flufsspat wird zur Entfernung darin befindlicher Schlackenteile gesiebt und kann dann wieder von neuem Verwendung finden. Mit einer fast wertlosen Blechbüchse und ungefähr $3 \mathrm{~kg}$ Flufsspat kann man sehr viele Thermitreaktionen ausführen. Statt des Flufsspats kann man natürlich in geeigneten Fällen auch Magnesiumoxyd, geschmolzenen und gepulverten Borax, Bortrioxyd, Natriummetaphosphat u. dgl. als Gefälsmaterial verwenden. Unser Verfahren hat auch noch den Vorteil, dal's man die Schlacken, die bei der Darstellung seltener Metalle, wie Vanadin, oft ziemlich beträchtliche Mengen wertvoller Bestandteile enthalten, durch einfaches Absieben vom Flufsspat so gut wie vollständig zur Regenerierung zurückerhält, während sie bei Verwendung fester Tiegel an die Tiegelwandungen angeschmolzen sind. Sollte einmal ein Versuch versagen, so dafs man genötigt ist, das noch pulverförmige Reaktionsgemenge aus dem Flufsspatschachte wieder herauszuholen, so schiebt man zuerst den Zylinder in den Schacht und trennt dadurch den Schachtinhalt von dem umgebenden Flufsspatpulver, das man nun leicht entfernen kann, ohne dals der Thermit damit vermischt wird.

Wir haben in der beschriebenen Weise in zahlreichen Versuchen ungefähr $500 \mathrm{~g}$ Vanadinmetall mit Hilfe von CalciumAluminiumthermit hergestellt; um aber die Brauchbarkeit unserer Versuchsanordnung auch für die Darstellung anderer Metalle darzutun, haben wir in gleicher Weise und mit gleich gutem Erfolge noch Mangan, Chrom, Eisen, Kobalt und Nickel dargestellt. Titan, Wolfram und Molybdän wurden nur in Gestalt kleiner Metallflitter oder kleiner Kügelchen erhalten, die in der umgebenden Schlackenmasse verteilt waren.

1 Die Zündkirschen stellten wir in der bekannten Weise her, indem wir ein Gemenge von 10 Teilen Aluminiumpulver, 40 Teilen Bariumsuperoxyd und 7 Teilen Kaliumchlorat mit Hilfe von Kollodinm zu Kugeln formten, in welche ein etwa $10 \mathrm{~cm}$ langes Stück Magnesiumband gesteckt wurde. Letzteres wurde mit einem Zündholz oder einer Bunsenflamme entzündet. 


\section{Experimentelles.}

Aus der zahlreichen Reihe unserer Versuche geben wir die folgenden, besonders charakteristischen als Belege wieder.

1. Versuch. Nach der Formel: $6 \mathrm{~V}_{2} \mathrm{O}_{5}+15 \mathrm{Ca}+10 \mathrm{Al}=12 \mathrm{~V}+$ $5\left(\mathrm{Al}_{2} \mathrm{O}_{3} .3 \mathrm{CaO}\right)$ wurde ein Gemisch von $100 \mathrm{~g} \mathrm{~V}_{2} \mathrm{O}_{5},{ }^{1} 55 \mathrm{~g}$ Ca und $24.7 \mathrm{~g} \mathrm{Al} \mathrm{zur}$ Entzündung gebracht, die Reaktion verlief sehr heftig unter Auswurf von Schlacke. Das weifsglühende Reaktionsprodukt war dünnflüssig. Der blasenfreie zusammenhängende Regulus löste sich leicht von der Schlacke ab; die Schlacke war sehr hart und krystallinisch (mit dunkeln nadelförmigen Krystallen in den Hohlräumen). - Gewicht des Regulus $37.5 \mathrm{~g}$, Ausbeute $66.8 \%$ \% - Vanadingehalt des Regulus $94.45 \%$.

2. Versuch. Wie beim 1. Versuche wurden $100 \mathrm{~g} \mathrm{~V}_{2} \mathrm{O}_{5}$ mit $55 \mathrm{~g} \mathrm{Ca}$ and $24.7 \mathrm{~g}$ Al gemischt und der Mischung noch $50 \mathrm{~g}$ grobgepulverten Vanadins mit viel anhaftender Schlacke von einem früheren Versuche zugesetzt. Die Reaktion verlief wieder sehr heftig, aber doch etwas rubiger als bei Versuch 1 . Am Boden der vollstïndig durchgesehmolzenen Reaktionsmasse befand sich ein blasenfreies rundlicher Regulus von $5 \mathrm{~cm}$ Durchmesser und $1 \mathrm{~cm}$ Dicke, der sich von der Schlacke leicht ablöste. Er zeigte auf dem Bruche krystallinische Struktur, wog $72 \mathrm{~g}$ und enthielt $93.8 \%$ Vanadin.

3. Versuch. Ausgeführt wie Versuch 1, aber mit anderem Calciummetall. Sehr heftige Reaktion; dichte, harte Schlacke. Drei kleinere Reguli im Gresamtgewichte von $35 \mathrm{~g}$. Ausbeute $62.3 \%$. Vanadingehalt des Metalls in zwei Proben 91.6 und $92.5 \%$.

4. Versuch. Auf den Grund des Fluorcalciumschachtes wurde eine Schicht geschmolzenen und gepulverten Chlornatriums eingestampft und darauf ein Gemisch von $200 \mathrm{~g} \mathrm{~V}_{2} \mathrm{O}_{5}, 110 \mathrm{~g} \mathrm{Ca}$ und $49.5 \mathrm{~g} \mathrm{Al}$ zur Entzündung gebracht. Die Reaktion verlief ungemein heftig. Infolge der Verdampfung von Chlornatrium flors das Metall nicht völlig zusammen, neben einem grofsen Regulus von knolliger Oberfäche wurden zahlreiche kleinere Metallkügelchen erhalten. Auf dem Bruche war das Metall ganz grobkrystallinisch und zeigte Hohlräume, die mit $\mathrm{NaCl}$ ausgekleidet waren. Vanadingehalt des Metalls $94.15 \%$. Die Ausbeute war schlecht und nicht genau zu bestimmen, da in der Schlacke sich noch zahlreiche Metallkügelchen befanden.

5. Versuch. Nach der Formel: $\mathrm{V}_{2} \mathrm{O}_{5}+2 \mathrm{Ca}+2 \mathrm{Al}=2 \mathrm{~V}+\mathrm{Al}_{2} \mathrm{O}_{5} \cdot 2 \mathrm{CaO}$ wurden $109.4 \mathrm{~g} \mathrm{~V}_{2} \mathrm{O}_{5}, 48.1 \mathrm{~g} \mathrm{Ca}$ und $32.5 \mathrm{~g} \mathrm{Al}$ gemischt; der Mischung wurden noch $76 \mathrm{~g}$ von früher erbaltenem Metall mit anhängender Schlacke, sowie $4 \mathrm{~g}$ $\mathrm{Ca}$ und $1.7 \mathrm{~g} \mathrm{Al}$ zugesetzt und dann das Ganze entzündet. Die Reaktion verlief ziemlich ruhig. Es wurde ein grofser blasenfreier Regulus von krystallinischem Bruche mit einem Vanadingehalt von $93.25 \%$ erhalten.

6. Versuch. Entsprechend der Formel: $2 \mathrm{Cr}_{2} \mathrm{O}_{3}+3 \mathrm{Ca}+2 \mathrm{Al}=4 \mathrm{Cr}+$ $\mathrm{Al}_{2} \mathrm{O}_{3} .3 \mathrm{CaO}$ wurde ein Gemenge von $60.9 \mathrm{~g} \mathrm{Cr}_{2} \mathrm{O}_{3}, 24 \mathrm{~g} \mathrm{Ca}$ und $10.8 \mathrm{~g}$ Al ent.

1 Bei allen Versuchen verwendeten wir Vanadinpentoxyd, das durch vorsichtiges Rösten von reinem Ammoniummetavanadat erhalten, dann geschmolzen und gepulvert worden war. Das durch Rösten erhaltene, nicht geschmolzene lockere, amorphe Pentoxyd wird zu leicht verstäubt. 
zündet. Die Reaktion verlief ganz ruhig, ohne Spritzen. Es wurde ein schöner Regulus von $31 \mathrm{~g}$ Gewicht erbalten. Ausbeute $74.3 \%$.

7. Versuch. Nach der Gleichung: $3 \mathrm{MnO}_{2}+3 \mathrm{Ca}+2 \mathrm{Al}=3 \mathrm{Mu}+\mathrm{Al}_{2} \mathrm{O}_{6} \mathrm{Ca}_{8}$ wurde ein Gemisch von $52.2 \mathrm{~g}$ Braunstein, $24 \mathrm{~g} \mathrm{Ca}$ und $10.8 \mathrm{~g}$ Al entzündet. In einer ziemlich heftigen Reaktion wurde ein Manganregulus von $15 \mathrm{~g} \mathrm{Ge-}$ wicht erhalten. Ausbeute $45.5 \%$.

8. Versuch. Nach der Gleichuvg: $6 \mathrm{CoO}+3 \mathrm{Ca}+2 \mathrm{Al}=6 \mathrm{Co}+\mathrm{Al}_{2} \mathrm{O}_{6} \mathrm{Ca}_{3}$ wurden $90 \mathrm{~g}$ Kobaltoxyd mit $24 \mathrm{~g} \mathrm{Ca}$ und $10.8 \mathrm{~g} \mathrm{Al}$ gemischt. Die Reaktion verlief ruhig. Der Kobaltregulus wog $32 \mathrm{~g}$. Ausbeute $45.2 \%$.

9. Versuch. $44.8 \mathrm{~g}$ Nickeloxyd wurden mit $12 \mathrm{~g}$ Calcium und $5.4 \mathrm{~g}$ Aluminiumgries gemengt, entsprechend der Gleichung: $6 \mathrm{NiO}+3 \mathrm{Ca}+2 \mathrm{Al}=$ $6 \mathrm{Ni}+\mathrm{Al}_{2} \mathrm{O}_{6} \mathrm{Ca}_{8}$. Dic Reaktion verlief ruhig und lieferte einen blasenfreien Nickelregulus. Gewicht $12 \mathrm{~g}$. Ausbeute $34.1 \%$.

München, Laboratorium für angewandte Chemie an der kgl. Universität, im Juni 1909.

Bei der Redaktion eingegangen am 2. Juli 1909. 